\title{
Jet quenching in heavy ion collisions at $\mathrm{LHC}^{1}$
}

\author{
I.P. Lokhtin ${ }^{\dagger}$ \\ M.V.Lomonosov Moscow State University, D.V.Skobeltsyn Institute of Nuclear Physics \\ $\dagger$ E-mail: igor@lav01.sinp.msu.ru
}

\begin{abstract}
We discuss the potential information about highly excited QCD-matter provided by mediuminduced partonic energy loss, known as "jet quenching". In particular, with its large acceptance hadronic and electromagnetic calorimetry, the Compact Muon Solenoid detector at LHC collider is a promising device to study these effects. We present physics simulations of observables such as the jet distribution with impact parameter, the azimuthal anisotropy of jet quenching, and the effects of $b$-quark energy loss on the high-mass dimuon continuum and secondary $J / \psi$ production.
\end{abstract}

\section{Hard probes at LHC with CMS detector}

High- $p_{T}$ QCD phenomena are perspective tools to study the properties of the hot matter created in ultrarelativistic nuclear interactions [1]. Hard probes such as quarkonia, hard jets and heavy quarks are not thermalized but carry information about the earliest stages of a system subject to numerous final-state effects in heavy ion collisions at SPS and RHIC. At the LHC, a new regime is reached where hard and semi-hard QCD multi-particle production can already dominate over underlying soft events.

The following signals of jet quenching due to medium-induced parton energy loss [2] can be identified as being observable in heavy ion collisions at LHC [1].

1) The suppression of high- $p_{T}$ jet pairs as compared to what is expected from independent nucleon-nucleon interactions pattern [3]. It includes modification of jet impact parameter dependence [4] and azimuthal anisotropy of jet quenching in non-central collisions [5].

2) The $p_{T}$-imbalance in $\gamma+$ jet $[6]$ and $Z\left(\rightarrow \mu^{+} \mu^{-}\right)+$jet [7] channels.

3) The suppression of high- $p_{T}$ hadron and photon (leading particle) yields due to a modification of the jet fragmentation function [8].

4) The modification of the high mass dimuon spectra from semileptonic $B$ and $D$ meson decays and secondary charmonium production due to heavy quark energy loss $[9,10]$.

All above signals can be studied in CMS Heavy Ion Programme [11]. The Compact Muon Solenoid (CMS) is a general purpose detector designed primary to search for the Higgs boson in proton-proton collisions at LHC [12]. Accordingly, the detector is optimized for accurate measurements of the characteristics of high-energy leptons, photons and hadronic jets in a large acceptance, providing unique capabilities for "hard probes" for both $p p$ and $A A$ collisions. The central element of CMS is the magnet, a $13 \mathrm{~m}$ long and $6 \mathrm{~m}$ diameter solenoid with an internal radius $\approx 3 \mathrm{~m}$, which will provide a strong $4 T$ uniform magnetic field. The $4 \pi$ detector consists of a 6 $\mathrm{m}$ long and $1.3 \mathrm{~m}$ radius central tracker, electromagnetic $(\mathrm{ECAL})$ and hadronic $(\mathrm{HCAL})$ calorimeters inside the magnet and muon stations outside. The tracker and muon chambers covers the pseudorapidity $|\eta|<2.4$, while the ECAL and HCAL calorimeters reach $|\eta|=3$. A pair of quartz-fiber very forward (HF) calorimeters, located $\pm 11 \mathrm{~m}$ from the interaction point, cover the region $3<|\eta|<5$ and complement the energy measurement. The tracker is composed of pixel layers and silicon strip counters. The CMS muon stations consist of drift tubes in the barrel region and cathode strip chambers in the end-cap regions. The electromagnetic calorimeter is made of almost 83000 scintillating $\mathrm{PbWO}_{4}$ crystals, and the hadronic calorimeter consists of scintillator inserted between copper absorber plates. The main characteristics of calorimeters are presented in Table 1.

Table 2 presents the event rates for various channels including hard jets in one month of $\mathrm{Pb}-\mathrm{Pb}$ beams assuming two weeks of data taking and luminosity $L=5 \times 10^{26} \mathrm{~cm}^{-2} \mathrm{~s}^{-1}$. The production cross sections in minimum bias $A A$ collisions were obtained from those in $p p$ interactions at the same energy, $\sqrt{s}=5.5 \mathrm{TeV}$, using simple parameterization $\sigma_{A A}^{h}=A^{2} \sigma_{p p}^{h}$. The $p p$ cross sections were evaluated using the PYTHIA_6.1 [13] with the CTEQ5L parton distribution functions. The estimated for the CMS acceptance statistics will be large enough to carefully study the dijet rate as a function of impact parameter as well as the $\varphi$ and $\eta$ distributions of jets. The statistics for the $\gamma+$ jet channel are satisfactory for studying the $E_{T}$-imbalance of the process, but the large background from jet+jet $\left(\rightarrow \pi^{0}\right)$ is still under investigation. The statistics for $Z\left(\rightarrow \mu^{+} \mu^{-}\right)+$jet channel are rather low, but the background is less than $10 \%$ in this case. Note that jet reconstruction in heavy ion collisions at CMS with efficiency and purity close to $100 \%$ is possible starting $E_{T}^{j e t} \sim 100 \mathrm{GeV}$ with energy resolution worser by factor $\sim 2$ as compared to $p p$ case [11].

${ }^{1}$ Talk given at XXXII International Symposium on Multiparticle Dynamics, Alushta, Crimea, September 7-13, 2002. 
Table 1: Energy resolution $\sigma / E=a / \sqrt{E} \bigoplus b$ and granularity of CMS calorimeters in barrel (HB, EB), endcap (HE, EE) and very forward ( $\mathrm{HF}$ ) regions. The energy resolution is shown for the total energy of $e$ and $\gamma(\mathrm{EB}$, EE) and transverse energy of hadronic jets (HB, HE, HF).

\begin{tabular}{|l|c|c|c|c|c|}
\hline Rapidity & \multicolumn{2}{|c|}{$0<|\eta|<1.5$} & \multicolumn{2}{|c|}{$1.5<|\eta|<3.0$} & $3.0<|\eta|<5.0$ \\
\hline Subdetector & HB & EB & HE & EE & HF \\
\hline$a$ & 1.16 & 0.027 & 0.91 & 0.057 & 0.77 \\
$b$ & 0.05 & 0.0055 & 0.05 & 0.0055 & 0.05 \\
\hline granularity & & & & $0.0174 \times 0.0174$ & \\
$\Delta \eta \times \Delta \varphi$ & $0.087 \times 0.087$ & $0.0174 \times 0.0174$ & $0.087 \times 0.087$ & to $0.05 \times 0.05$ & $0.175 \times 0.175$ \\
\hline
\end{tabular}

Table 2: Expected rates for various channels including hard jets in one month of $\mathrm{Pb}-\mathrm{Pb}$ beams.

\begin{tabular}{|l|c|c|}
\hline Channel & Barrel & Barrel+Endcap \\
\hline jet + jet, $E_{T}^{\text {jet }}>100 \mathrm{GeV}$ & $2.1 \times 10^{6}$ & $4.3 \times 10^{6}$ \\
\hline$\gamma+$ jet, $E_{T}^{\text {jet }, \gamma}>100 \mathrm{GeV}$ & $1.6 \times 10^{3}$ & $3.0 \times 10^{3}$ \\
\hline$Z\left(\rightarrow \mu^{+} \mu^{-}\right)+$jet, $E_{T}^{\text {jet }}, P_{T}^{Z}>100 \mathrm{GeV}$ & 30 & 45 \\
\hline
\end{tabular}

\section{Jets versus impact parameter and azimuthal angle}

We have analyzed the impact parameter dependence of jet rates in $\mathrm{Pb}-\mathrm{Pb}$ collisions at $\mathrm{LHC}$ (see Ref. [4] for model details). Figure 1 shows dijet rates in different impact parameter bins $\left(E_{T}^{\text {jet }}>100 \mathrm{GeV},\left|\eta^{\text {jet }}\right|<2.5\right)$ : (i) without energy loss, (ii) with collisional loss only, (iii) with collisional and radiative loss from BDMS model [14]. The bin width $2 \mathrm{fm}$ in Fig. 1 corresponds to estimated accuracy of impact parameter determination using correlation between $b$ and energy flow deposited in very forward CMS HF calorimeter [15]. The maximum and mean values of $d N^{\text {dijet }} / d b$ distribution get shifted towards the larger $b$ because jet quenching is stronger in central collisions than in peripheral one's,. Since the coherent LPM radiation induces a strong dependence of the radiative energy loss of a jet on the angular cone size $[16,17]$, the result for jets with non-zero cone size $\theta_{0}$ is expected to be between $(i i i)\left(\theta_{0} \rightarrow 0\right)$ and $(i i)$ cases. Thus the observation of a dramatic change in the $b$-dependence of jet rates as compared to what is expected from independent nucleon-nucleon interactions pattern, would indicate the existence of medium-induced parton rescattering.

Other interesting feature of jet production in semi-central $A A$ collisions can be azimuthal anisotropy of high$p_{T}$ hadrons $[18,19]$ or jets [5] due to parton energy loss in azimuthally non-symmetric volume of quark-gluon plasma. Figure 2 shows the distribution of jets over azimuthal angle $\varphi$ with collisional and radiative loss (a) and collisional loss only (b) for $b=0,6$ and $10 \mathrm{fm}$. The same conditions as for Fig. 1 are fulfilled. The plot is normalized on the distributions of jets over $\varphi$ in $\mathrm{Pb}-\mathrm{Pb}$ collisions without energy loss. The azimuthal anisotropy gets stronger as going from central to semi-central collisions, but the absolute suppression factor reduces with increasing $b$. The methodical advantage of azimuthal jet observables is that one needs to reconstruct only azimuthal position of jet without measuring total jet energy. The summarized in papers [20] methods for determination of the event plane are applicable for studying anisotropic flow in current heavy ion experiments at SPS and RHIC, and might be also used at LHC [5]. It was also suggested recently in [21] the method for measurement of jet azimuthal anisotropy without reconstruction of the event plane. This technique is based on the calculation of correlations between the azimuthal position of jet axis and the angles of particles (not incorporated in the jet) using as weights the particle momenta or energy deposition in the calorimetric sectors.

\section{Heavy quarks: $B \bar{B} \rightarrow \mu^{+} \mu^{-}$and $B \rightarrow J / \psi$ modes}

The open heavy flavour production is important due to opportunity to study the massive colour charge in quarkgluon environment, while jets probe the behaviour of massless partons in QCD-matter. The charm and bottom production cross section are much larger at LHC as compared with RHIC. Thus the systematical studies of heavy flavour (especially $B$ ) physics can be performed in CMS. It was found in the high invariant mass dimuon range 10 $\mathrm{GeV} / \mathrm{c}^{2} \leq M \leq 70 \mathrm{GeV} / \mathrm{c}^{2}$ the dominant contribution comes from $B \bar{B}$ fragmentation, and its extraction from uncorrelated and correlated background is possible [11, 10]. In-medium gluon radiation and collisional energy loss of heavy quarks can result in suppression and modification of high mass dilepton $[9,10]$ and secondary $B \rightarrow J / \psi[10]$ spectra. In addition, finite quark mass effects can lead to the suppression of medium-induced radiation of heavy quarks as compared with massless partons, enhancing e.g. the $B / \pi$ ratio [22]. 


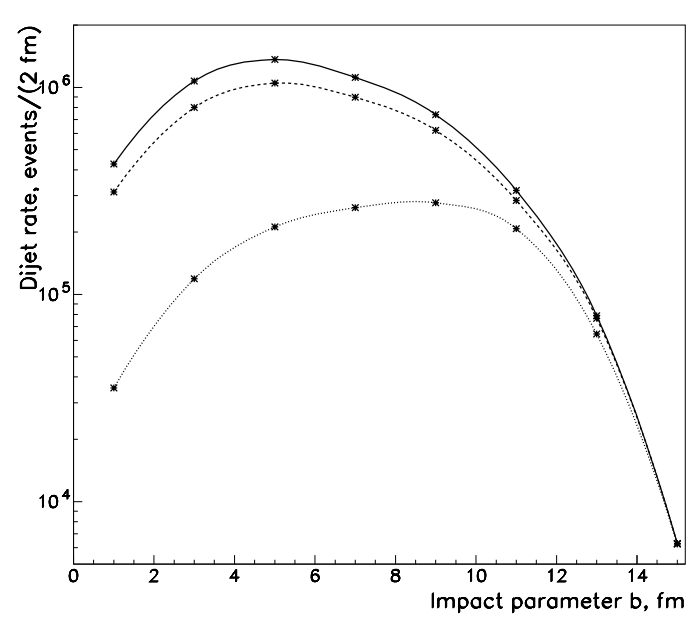

Figure 1: The dijet rates vs. impact parameter $b$ : without energy loss (solid curve), with collisional loss (dashed), with collisional and radiative loss (dotted).
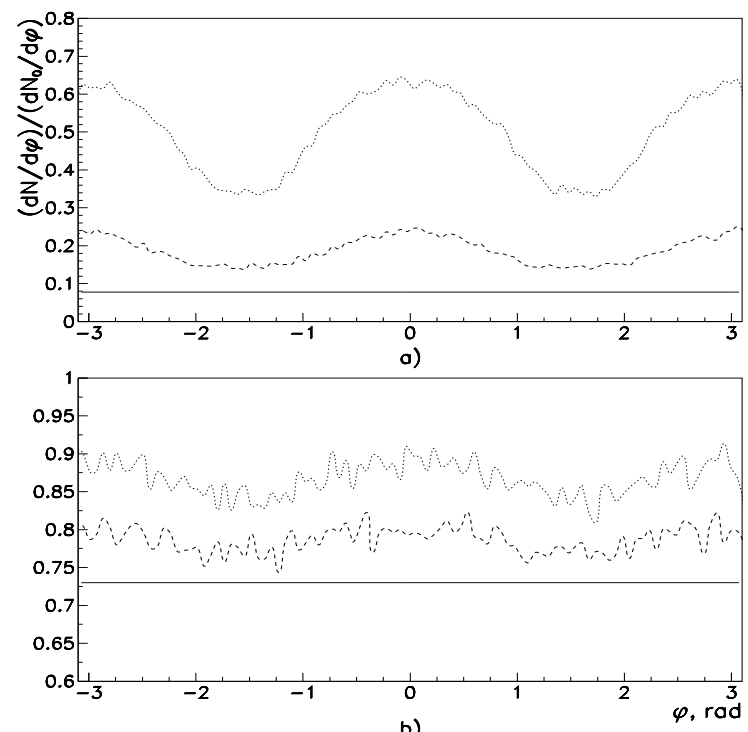

Figure 2: The distribution of jets over azimuthal angle: collisional and radiative loss (a), collisional loss only (b). From bottom to top: $b=0,6$ and $10 \mathrm{fm}$.

Invariant mass distribution of $\mu^{+} \mu^{-}$in $\mathrm{Pb}-\mathrm{Pb}, \sqrt{ } \mathrm{s}=5.5 \mathrm{~A} \mathrm{TeV}$
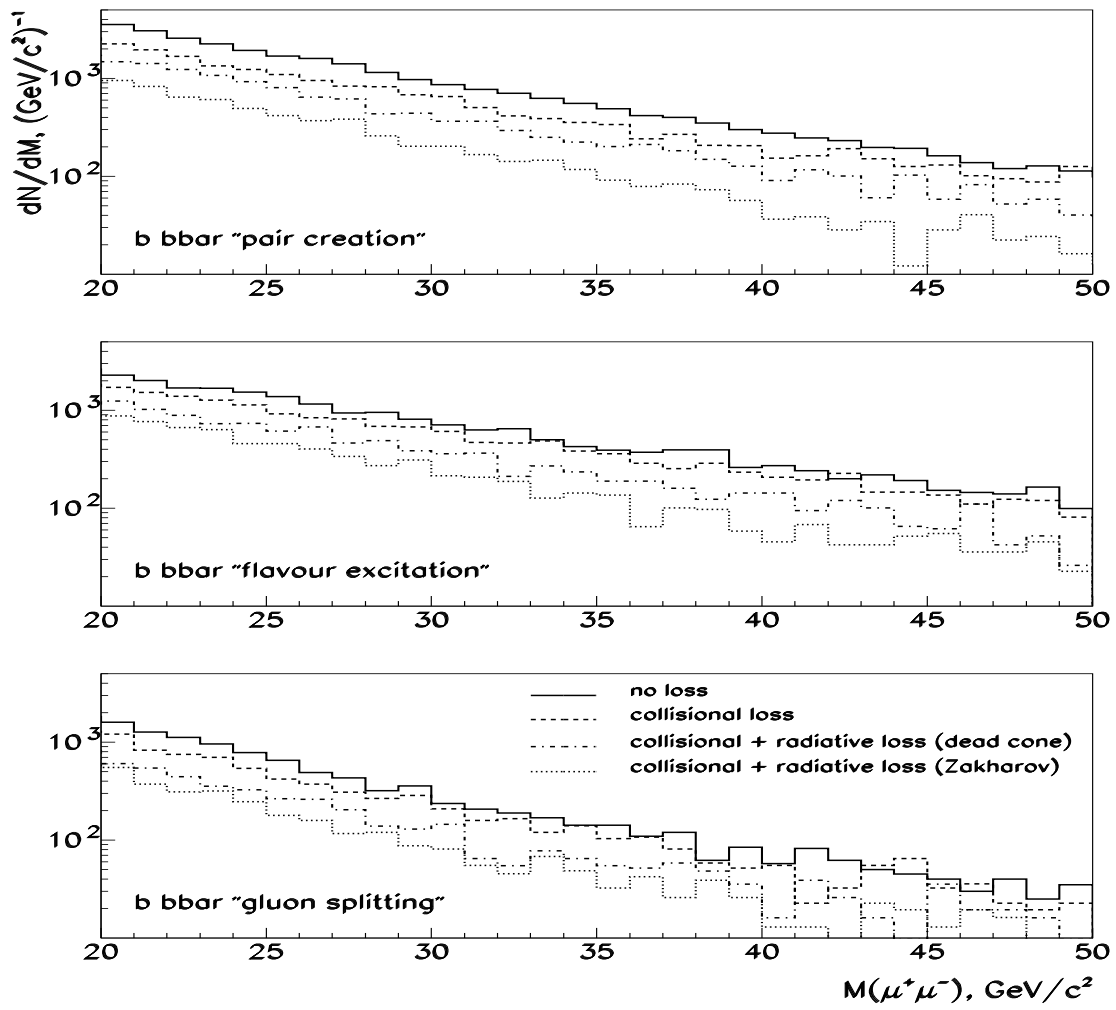

Figure 3: Invariant mass distribution of $\mu^{+} \mu^{-}$pairs from $B \bar{B}$ decays for several models of energy loss. The muon requirements are $p_{T}^{\mu}>5 \mathrm{GeV} / \mathrm{c}$ and $\left|\eta^{\mu}\right|<5$. 
The important predicted feature of heavy quark production at LHC is that the contribution of gluon splittings in initial- or final-state shower evolution to heavy flavour yield can be important [23], while at present accelerator energies the most of heavy quarks being produced due to direct hard scatterings ("pair creation"). These are so-called "flavour excitation" (one heavy quark is produced in vertex of hard process and another quark is created from initial state parton shower) and "gluon splitting" (both heavy quarks are produced from final state parton shower). It was shown in [24] that such "showering" $b \bar{b}$ pairs are an important contribution to the dilepton continuum at high masses and, in fact, are of the same order as direct $q \bar{q} \rightarrow Q \bar{Q} X$ and $g g \rightarrow Q \bar{Q} X$ pair creation at the LHC for dimuons with masses greater than $10 \mathrm{GeV} / \mathrm{c}^{2}$. Moreover, most of secondary $J / \psi$ 's produced in $B$ decays originate from the $g g \rightarrow g\left(g^{*} \rightarrow Q \bar{Q}\right)$ diagram [24].

Figure 3 shows the dimuon invariant mass spectra at CMS acceptance for several models: without loss; with rescattering and collisional loss; with rescattering, collisional and radiative loss calculated as incoherent limit of Zakharov's formula [25], and also from "dead-cone" model [22]. The suppression factor $\sim 2-4$ can be clearly observed over "nuclear shadowing" effect [26], $\sim 15 \%$. Estimated suppression by a factor of $\sim 1.5-2$ for secondary $J / \psi$ is less than for $B \bar{B}$ decays and is comparable with the shadowing corrections $(\sim 30 \%)$.

\section{Conclusion}

To summarize, with its large acceptance, almost hermetic hadronic and electromagnetic calorimetry of fine granularity and good energy and spatial jet resolution, and excellent muon capabilities, the Compact Muon Solenoid (CMS) detector is a promising device to study jet quenching at LHC in various channels. Jet reconstruction in heavy ion collisions at CMS is possible starting $E_{T}^{\text {jet }}=100 \mathrm{GeV}$, and estimated statistics will be large enough to carefully study the jet rate as a function of impact parameter, as well as azimuthal and rapidity distributions of jets. Impact parameter and event plane determination are possible using energy flow measurements. In-medium energy loss of heavy quarks also can be observable in $B \bar{B} \rightarrow \mu^{+} \mu^{-}$and $B \rightarrow J / \psi \rightarrow \mu^{+} \mu^{-}$modes. However, in order to determine the baseline rates precisely, measurements in $p p$ or $d d$ collisions at the same or similar energies per nucleon as in the heavy ion runs are strongly desirable.

Acknowledgements. I would like to thank the organizers of the Symposium for the warm welcome and stimulating atmosphere. Discussions with M. Bedjidian, D. Denegri, Yu.L. Dokhitzer, D. Kharzeev, O.L. Kodolova, A.N. Nikitenko, S.V. Petrushanko, L.I. Sarycheva, S.V. Shmatov, A.M. Snigirev, V.V. Uzhinskii, I.N. Vardanian, S.A. Voloshin, R. Vogt, U. Wiedemann, P.I. Zarubin and G.M. Zinoviev are gratefully acknowledged.

\section{References}

[1] CERN Workshop "Hard Probes in Heavy Ion Collisions at the LHC", http://wwwth.cern.ch/lhcworkshop/lhcworkshop01.html .

[2] R. Baier, D. Schiff and B.G. Zakharov, Annual Rev. Nucl. Part. Sci. 50, 37 (2000).

[3] M. Gyulassy and M. Plümer, Phys. Lett. B 243, 432 (1990).

[4] I.P. Lokhtin and A.M. Snigirev, Eur. Phys. J. C 16, 527 (2000).

[5] I.P. Lokhtin et al., e-print hep-ph/0112180; Phys. At. Nucl. 65, 943 (2002).

[6] X.-N. Wang, Z. Huang and I. Sarcevic, Phys. Rev. Lett. 231, 77 (1996).

[7] V. Kartvelishvili, R. Kvatadze and R. Shanidze, Phys. Lett. B 356, 589 (1995).

[8] M. Gyulassy and X.-N. Wang, Phys. Lett. B 68, 1480 (1992).

[9] Z. Lin and R. Vogt, Nucl. Phys. B 544, 339 (1999).

[10] I.P. Lokhtin and A.M. Snigirev, J. Phys. G 27, 2365 (2001).

[11] G. Baur et al., Heavy Ion Physics Programme in CMS, CERN CMS Note 2000/060.

[12] CMS Collaboration Technical Proposal, CERN/LHCC 94-38, 1994.

[13] T. Sjöstrand, Comput. Phys. Commun. 82, 74 (1994).

[14] R. Baier et al., Nucl. Phys. B 531, 403 (1998).

[15] I. Damgov et al., Part. Nucl. Lett. 107, 93 (2001); CERN CMS Note 2001/055.

[16] R. Baier et al., Phys. Rev. C 58, 1706 (1998).

[17] I.P. Lokhtin and A.M. Snigirev, Phys. Lett. B 163, 440 (1998).

[18] X.-N. Wang, Phys. Rev. C 63, 054902 (2001).

[19] M. Gyulassy, P. Levai and I. Vitev, Nucl. Phys. B 594, 371 (2001).

[20] S.A. Voloshin and Y. Zhang, Z. Phys. C 70, 665 (1996); A.M. Poskanzer and S.A. Voloshin, Phys. Rev. C 58, 1671 (1998).

[21] I.P. Lokhtin, L.I. Sarycheva and A.M. Snigirev, Phys. Lett. B 537, 261 (2002).

[22] Yu.L. Dokshitzer and D. Kharzeev, Phys. Lett. B 519, 199 (2001).

[23] E. Norrbin and T. Sjöstrand, Eur. Phys. J. C 17, 137 (2000).

[24] I.P. Lokhtin, A.M. Snigirev, Nucl. Phys. A 702, 34 (2002).

[25] B.G. Zakharov, JETP Lett. 65, 615 (1997); Phys. At. Nucl. 61, 838 (1998).

[26] K.J. Eskola, V.J. Kolhinen and C.A. Salgado, Eur. Phys. J. C 9, 61 (1999). 